\title{
Concomitant emphysematous cystitis and liver abscess
}

\author{
Chih-Cheng Lai
}

Department of Intensive Care Medicine, Chi Mei Medical Center, Tainan, Taiwan
Received: July 28, 2015

Revised : August 7, 2015

Accepted: February 13, 2017

\section{Correspondence to}

Chih-Cheng Lai, M.D.

Tel: +886-6-622-6999 (ext. 359)

Fax: +886-6-622-361

E-mail:dtmed141@gmail.com
A 31-year-old man with a fever and abdominal pain for 3 days presented to the emergency department. Upon arrival, his vital signs were temperature $38.2^{\circ} \mathrm{C}$, pulse rate 131 beats $/ \mathrm{min}$, respiratory rate 18 breaths/min, and blood pressure 138/63 mmHg. Physical examinations revealed icteric sclera, right upper quadrant (RUQ) tenderness elicited on deep palpation, and suprapubic region tenderness. Laboratory examination results were white blood cell (WBC) count 19,800/mm³, platelet count $58,000 / \mathrm{mm}^{3}$, glucose 730 $\mathrm{mg} / \mathrm{dL}$, creatinine $3.9 \mathrm{mg} / \mathrm{dL}$, aspartate aminotransferase $754 \mathrm{IU} / \mathrm{L}$, total biliru-
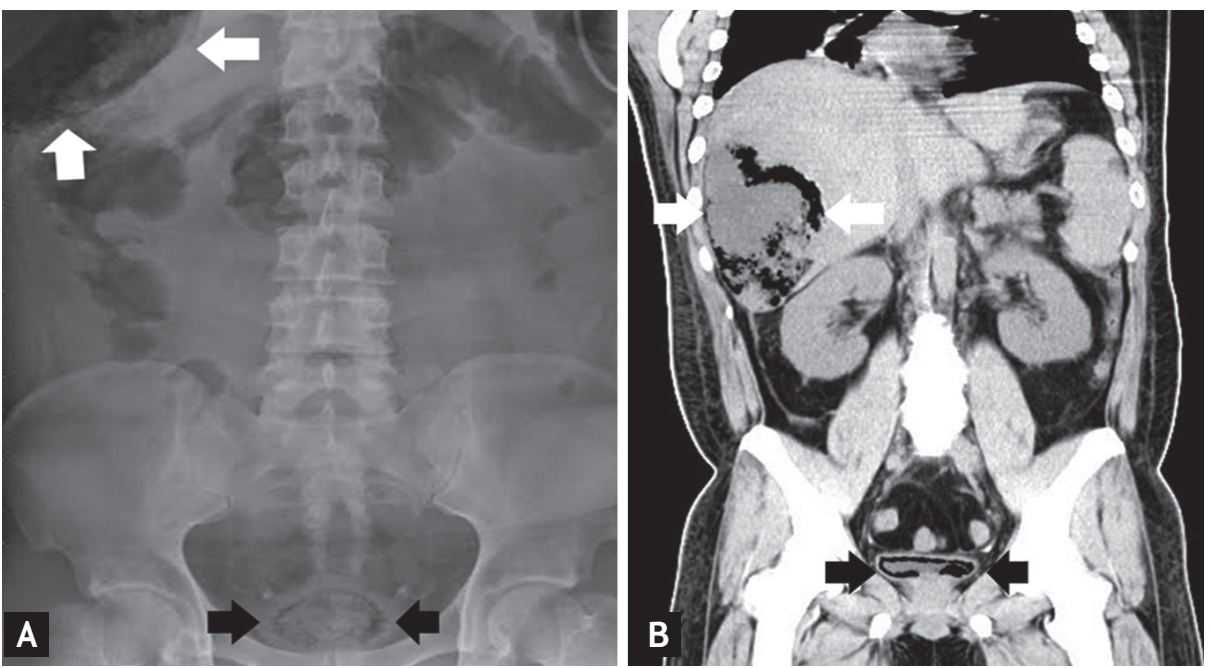

Figure 1. (A) Radiography of the abdomen revealed a mottled collection of gas over the right upper quadrant (white arrows), and beaded necklace-like air over the urinary bladder area (black arrows). (B) Computed tomography of the abdomen disclosed a large (19.0 $\times 10.0 \times 17.7 \mathrm{~cm}$ ) gas-producing liver lesion (white arrows), intra-luminal air within the wall of the urinary bladder (black arrows), and the smooth contours of the bilateral kidneys, which showed no evidence of infection, e.g., fat stranding and air content. 
patient was treated empirically with a parenteral antibiotic (flomoxef: 1 g every 8 hours) for 28 days, percutaneous drainage of his liver abscess, and insulin to control his blood sugar. Three days later, two sets of blood cultures, drained pus, and a urine specimen grew Klebsiella pneumoniae, which was resistant only to ampicillin, but susceptible to amikacin, ciprofloxacin, ceftazidime, flomoxef, cefuroxime, cefazolin, gentamicin, and piperacillin/tazobactam. After 1 month, his clinical condition stabilized, he was discharged on a regimen of oral cefixime (100 mg three times daily) for 2 weeks, and his 2-month outpatient follow-up was uneventful.

Emphysematous cystitis is a gas-forming infection of the bladder wall [1]. It is caused most commonly by Escherichia coli, Enterobacter aerogenes, and K. pneumoniae. Concomitant K. pneumoniae emphysematous cystitis and a liver abscess is rare, and the mechanism involved in this case might be that initially $K$. pneumoniae caused a liver abscess, and then spread hematogenously to cause bacteremia and additional complications [2]. In addition, a K. pneumoniae liver abscess might be caused by an initial colonization of the colon which then reaches the liver via the portal venous system. Although CT is the diagnostic modality of choice for a liver abscess and for emphysematous cystitis, an X-ray of the kidney-ureter-bladder region might be sufficient for a diagnosis. Appropriate antibiotic therapy and adequate drainage are essential for treating this disorder.

\section{Conflict of interest}

No potential conflict of interest relevant to this article was reported. 